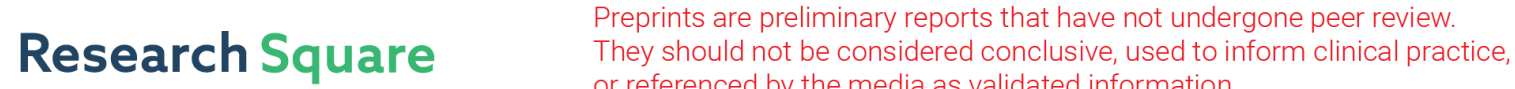 or referenced by the media as validated information. \\ REBACIN® is an optional intervention for persistent high-risk human papillomavirus infection: a retrospective analysis of 364 patients
}

\section{Ying Yang}

Sichuan University West China Second University Hospital

Ting Wen Yi Hu

Sichuan Univeraity West China Second University Hospital

\section{Xiu Ming}

Sichuan University West China Second Hospital

\section{E Yang}

Sichuan University West China Second University Hospital

Zhengyu Li ( $\nabla$ zhengyuli@scu.edu.cn )

Sichuan University West China Second University Hospital

Research article

Keywords: Human papillomavirus, HPV, REBACIN®, therapeutic efficacy

Posted Date: May 7th, 2020

DOl: https://doi.org/10.21203/rs.3.rs-25454/v1

License: (c) (i) This work is licensed under a Creative Commons Attribution 4.0 International License. Read Full License

Version of Record: A version of this preprint was published at International Journal of Gynecology \& Obstetrics on October 13th, 2020. See the published version at https://doi.org/10.1002/ijgo.13385. 


\section{Abstract}

Background: Persistent high-risk human papillomavirus (hrHPV) infection is a crucial cause of cervical cancer, for which optimal pharmacological intervention remains unavailable. This study aimed at evaluating the therapeutic efficacy of REBACIN® in patients with persistent hrHPV infection.

Methods: This study is a combination of a retrospective analysis and a meta-analysis. The retrospective analysis included 364 patients who were persistently infected with HPV for at least twelve months, between September 2015 and February 2019, and only received REBACIN® intervention. HPV DNA typing, HC2 hrHPV DNA, and ThinPrep cytologic tests were performed before and after REBACIN $\circledast$ intervention, to evaluate the therapeutic efficacy. The meta-analysis included trials evaluating the therapeutic efficacy of interferons.

Results: After a follow-up period of three to six months, the overall effective rate of REBACIN ${ }^{\circledR}$ was $74.73 \%(272 / 364)$, which was higher than that of interferon (61.50\%). The efficacy of REBACIN® was correlated with HPV type (OR: 0.549, 95\% Cl: 0.367-0.822, P < 0.05) and pretreatment cytology (OR: $0.358,95 \%$ Cl: $0.173-0.739, \mathrm{P}<0.05)$.

Conclusions: REBACIN® is potently efficacious at clearing persistent hrHPV infection; hence, it can serve as an optional intervention for persistent hrHPV infection.

\section{Background}

Human papillomavirus (HPV) infection is the most pervasive sexually transmitted disease. Annually, there are over 14 and 45 million new cases diagnosed respectively in the United States [1] and China [2]. It is estimated that nearly $80 \%$ of the sexually active women are infected by HPV by the age of fifty years [3]. In most cases, the infection normally regresses within twenty-four months, even in the case of infection with high-risk HPV (hrHPV) genotypes which were proven to be closely correlated with cervical cancer. Among them, $50 \%$ were reportedly caused by HPV $16,20 \%$ by HPV 18 , and $19 \%$ by HPV $31,33,45$, 52 , or $58[4,5]$.

Worldwide, cervical cancer is the fourth most common cancer among women. Yearly, about 530 thousand cases are diagnosed and 260 thousand deaths are recorded. Persistent hrHPV infections will increase the risk of precancerous and cancerous lesions, especially when over twelve months. Additionally, a study showed that women with persistent hrHPV infections would either be free of infection or develop cervical intraepithelial neoplasia (CIN) $2+$ within six years, whatever with or without intensive clinical follow-up. The mean time of progression from the precancer stage to invasive cancer stage has been reported to be ten years [6-8]. Therefore, more efficient interventions for persistent hrHPV infections are needed to prevent progression.

On the basis of cytology and HPV test results, we can assess the risk of cervical cancer and determine the novt ctan for mananina it Traditinnal intorvantinnc fnr HPV infection mainly include routine screening, Loading [MathJax]/jax/output/CommonHTML/fonts/TeX/fontdata.js 
cryotherapy, laser ablation, cervical conization, loop electrosurgical excision procedure, therapeutic HPV vaccine, immunotherapy (e.g., imiquimod and interferon), and antiviral drugs (e.g., cidofovir). These methods have their indications and limits as well as benefits and side effects. However, the therapeutic efficacy of current interventions remains unsatisfactory, especially for early-stage pharmacotherapy, and many interventions are still in the clinical trial phase.

REBACIN ${ }^{8}$, as an antiviral biologic, can inhibit the expression of oncogenes $E 6$ and $E 7$, which play a crucial role in malignancy development, via the interactions of their respective protein products with the p53 tumor suppressor and retinoblastoma. In addition, it is known to effectively inhibit and clear herpes simplex virus infections, and its therapeutic efficacy in HPV is worth expecting. This study was aimed at evaluating the therapeutic efficacy of REBACIN® in patients with persistent hrHPV infection.

\section{Methods}

\section{Retrospective study}

\section{Study design and participants}

This study was approved by the Institutional Ethics Committee of West China Second University Hospital. Between September 2015 and February 2019, 364 patients continuously infected with HPV for at least twelve months and only treated with REBACIN® were retrospectively analyzed. The inclusion criteria were as follows: (1) sexually active women and (2) persistent hrHPV infection for more than twelve months. The exclusion criteria were as follows: (1) women who did not complete the designed administration of conventional drugs (REBACIN $\circledast$ was recommended to be used at least twenty days per month for three consecutive months); (2) women with a fundamental immune deficiency (such as after chemotherapy); (3) women with severe heart, lung, liver, or kidney dysfunction; (4) women with drug allergy; and (5) pregnant or lactating women.

The participants were followed-up for three to six months after their final drug administration, as the outcome of HPV DNA typing or HC2 hrHPV DNA tests before and after REBACIN® intervention were necessary. Besides, the results of the ThinPrep cytologic test (TCT) and colposcopy can aid efficacy determination.

\section{Test methods}

\section{Cervical cytology}

TCT, based on specimens obtained from the external surface of the ectocervix and endocervix, was performed for most of the participants, to detect cervical cell changes. It can identify abnormal cells from the squamocolumnar junction, where cervical dysplasia and cancers mostly develop. The results were reported using the terms in the Bethesda 2014 guidelines [9]. 
The HPV DNA typing test using the Luminex200 system was based on the principles of flow cytometry. It can be used to analyze 17 types of hrHPV (HPV 16, 18, 26, 31, 33, 35, 39, 45, 51, 52, 53, 56, 58, 59, 66, 68, and 82), including the HPV types confirmed to be closely related to cervical cancer by the American Society for Colposcopy and Cervical Pathology (ASCCP) in 2012 [10].

HC2 hrHPV DNA test: The HC2 hrHPV DNA test using the Hybrid-Capture 2 (HC2) technology was based on a nucleic acid hybridization assay for the qualitative detection of 13 types of hrHPV DNA in cervical specimens (HPV 16, 18, 31, 33, 35, 39, 45, 51, 52, 56, 58, 59, and 68). A relative light unit (RLU) measurement equal to or greater than the cutoff (CO) value indicates the presence of hrHPV DNA sequences, whereas the reverse indicates the absence of it in the specimen (positive for HPV infection: $\mathrm{RLU} / \mathrm{CO} \geq 1$; negative for HPV infection: RLU/CO < 1) [11].

\section{Evaluation of REBACIN@ efficacy}

With regard to the results of HPV DNA typing test, the effective rate was defined as the rate of clearance of more than one HPV type, whereas with regard to the results of the HC2 hrHPV DNA test, it was defined as the rate at which more than $25 \%$ of hrHPV load decreased [12-15].

\section{Statistical analyses}

The data were analyzed using SPSS software version 22.0. The counting data were analyzed via the chisquare test. All the $p$-values reported were two-sided, and p-values $<0.05$ indicated statistical significance. Moreover, binary logistic regression analysis was used to identify the major factors affecting the efficacy of REBACIN®.

\section{Meta-analysis}

\section{Search strategy and study selection}

We used the terms "(interferon OR interferon-a2b OR recombinant human $a-2 b$ interferon capsule for vaginal foams OR Xinfuning), (human papillomavirus OR HPV), (effectiveness OR efficacy OR effect), and (cervical)" to search PubMed, the Cochrane Library, Embase, and China National Knowledge Infrastructure (CNKI) from inception to December 2019. Studies that met the following criteria were included: (1) studies on cervical HPV infection; (2) studies on interferon intervention; and (3) studies with a follow-up period of three to six months.

\section{Data extraction and risk of bias}

The initial screening of the databases for study selection was based on information in the title and abstract, and it was conducted independently by two reviewers (Y.Y. and T.W.Y.H.). In cases of insufficient data, we reviewed the full text of the article for further information and extracted the author, year, location, study design, duration of follow-up, and sample size. The quality of the studies was assessed independently by two reviewers (Y.Y. and T.W.Y.H.), and discrepancies were resolved by consensus. 
We calculated the effective rate using DerSimonian-Laird weights for the random-effects model with Stata software version 15.0 .

\section{Results}

Our study had 364 participants whose general characteristics are listed in Table 1 . The mean age of the patients was $41.8 \pm 9.8$ years (range, $20-71$ years; standard deviation, 9.80 ) and most of them were in the $31-40$ and $41-50$ age groups.

Infection with single- and multi-type HPV was detected in 229 (62.91\%) and 97 (26.65\%) patients, respectively. HPV 16 was the most common genotype. Meanwhile, we collected the pretreatment cytology data of the patients and found that most of them had tested cytology-negative but were HPV-positive. A course is equivalent to regular use of REBACIN® for three consecutive months; most patients received one course (294/364) and a few received two or three courses. During the treatment, 162 patients were celibate, 56 had sex with a condom, and 55 had sex without a condom. Two hundred forty-one patients had a history of interferon therapy, which had no effect.

\section{The therapeutic efficacy of REBACIN@ compared with interferon}

The initial search identified 517 articles from electronic databases (PubMed, $n=162$; Cochrane, $n=44$; EMBASE, $n=290$; and CNKI, $n=21)$, after excluding duplications $(n=87)$, screening titles and abstracts $(n=378)$, and screening the full text $(n=52)$. Finally, we used six studies that met all the inclusion criteria (two RCTs and four observation studies), the studies were from two countries: 1 Mexico [16], 5 China [12, 17-20].

These studies included 303 patients, and the pooled effective rate of interferon was $61.50 \%(95 \% \mathrm{Cl}$, $0.480-0.749$ ). The results are presented in Table 2 and Fig. 1 . To objectively evaluate the therapeutic efficacy of REBACIN ${ }^{\circledR}$, we compared REBACIN® with interferon, currently considered efficient. In our retrospective study, the overall effective rate of REBACIN® was $74.73 \%(272 / 364)$, higher than that of interferon (61.50\%).

Additionally, Chen et al. [21] had shown that the self-clearance rate of hrHPV infection within six months was $35.6 \%$ (47/132). Further, a recent study by Yang et al. [14] showed that the self-clearance rates in three months of follow-up were $12.5 \%(8 / 64)$ and $20.0 \%(8 / 40)$. All results demonstrated that REBACIN ${ }^{8}$ is highly efficacious at clearing persistent hrHPV infection.

\section{Main factors affecting REBACIN® efficacy}

The results of the binary logistic regression analysis (Table 3 ) indicated that the therapeutic efficacy of REBACIN ${ }^{\circledR}$ was influenced by HPV type $(\mathrm{OR}: 0.549,95 \% \mathrm{Cl}$ : $0.367-0.822, \mathrm{P}<0.05)$ and pretreatment 
cytology (OR: $0.358,95 \% \mathrm{Cl}: 0.173-0.739, \mathrm{P}<0.05)$. However, there were no significant differences in age, course, sex life, and history of interferon treatment.

The results of the chi-square tests (Table 4) showed that REBACIN $®$ had a higher effective rate against multi-type than against single-type HPV infection $\left(x^{2}=20.21, P<0.01\right)$, whereas there were no significant differences in the effective rate between the three groups (HPV 16, HPV 18, and other single-type) $\left(x^{2}=\right.$ $1.08, P>0.05)$. REBACIN ${ }^{\circledR}$ was more effective when the results showed atypical squamous cells of undetermined significance (ASCUS) or low-grade squamous intraepithelial lesion (LSIL; $x^{2}=11.41, \mathrm{P}<$ 0.01) than when the cytology test results were negative.

\section{Nearly no adverse events}

A majority of the participants completed the treatment and follow-up visit, and only two reported vaginal itching. No other side effects were reported.

\section{Discussion}

A quantity of studies focused on the time of HPV regression and put forward the following: Approximately $56 \%$ of the women with persistent hrHPV infections remained positive after a year of follow-up, and the mean time for clearance of hrHPV infection was close to sixteen months $[16,17]$. However, hrHPV infections over twelve months increase the possibility of precancerous and cancerous lesions, and not all HPV infections can regress by natural immunity. Additionally, HPV can reactivate from a latent state in some populations. In that case, it is critical to clear the virus [22-24]. If pharmacotherapy is beneficial, an appropriate medicine is needed. In our study, the overall effective rate was $74.73 \%$ (272/364), within the six-month follow-up period, showing that REBACIN® markedly increased the HPV clearance rate and shortened persistent infection time, which was highly significant in preventing disease progression.

Cervical infections with hrHPV types could increase the risk of CIN2 + or worse, and infection with HPV 16 is associated with the highest risk of progression to CIN3+. In addition, it has the lowest chance and the longest clearance time (mean: 18.3 months) $[25,26]$. In our study, the effective rate of HPV 16 reached $60.78 \%(31 / 51)$ within six months, showing a significant effect, although it was lower than that associated with other types.

Patient aged $\geq 30$ years with positive HPV and negative cytology test are reportedly at an increased risk of cervical disease. Specifically, when a patient tests positive for an HPV 16 or 18 infection, a colposcopy should be performed immediately [27]. Katki et al. [28] also indicated that in 965,360 women, LSIL resulted in a five-year CIN3 + risk in $5.2 \%$, but $6.8 \%$ were HPV-positive/cytology-negative; the latter were at a higher risk of $\mathrm{CIN} 3+$. In our study, the pretreatment cytology test was an influential factor in determining efficacy, and ASCUS or LSIL seemed to have a higher effective rate than the negative one. More attention should be paid to patients with HPV-positive/cytology-negative results, and these patients should be 
In our study, although sex with or without condoms showed no significant differences, it was recommended that a condom be used while on medication. Correct and consistent condom use will reduce the risk of HPV infections [29]. We found that people who have sex without condoms might be associated with a slightly higher recurrence risk or may easily acquire a new hrHPV infection. This is evident by the fact that 14 of the 55 patients who did not use condoms had a recurrent or new infection, whereas only 23 of the 218 patients who did not have sex or had sex with condoms developed a recurrent or new infection.

As most patients in this study chose REBACIN® intervention (241/272) after ineffective interferon therapy, we collected information on their interferon therapy history, which showed that REBACIN ${ }^{\circledR}$ efficacy was uninfluenced by previous use or non-use of interferon. In other words, REBACIN® showed significant efficacy even in patients in whom interferon therapy was ineffective.

As for the limitations of our study, it was a non-randomized, -controlled trial, which yielded reliable results. Although we did not have a control group for many other studies on the effective rate of interferon have already been performed, we had an adequate number of participants and conducted a combined retrospective study and meta-analysis, ensuring result reliability.

\section{Conclusions}

In conclusion, the present study indicates that REBACIN® is potently efficacious at clearing persistent hrHPV infections and could serve as an optional intervention for persistent hrHPV infection.

\section{Abbreviations}

hrHPV: High-risk human papillomavirus

HPV: Human papillomavirus

CIN: Cervical intraepithelial neoplasia

TCT: ThinPrep cytologic test

ASCCP: American Society for Colposcopy and Cervical Pathology

HC2: Hybrid-Capture 2

ASCUS: Atypical squamous cells of undetermined significance

LSIL: Low-grade squamous intraepithelial lesion

\section{Declarations}


This retrospective study was approved by the Institutional Ethics Committee of West China Second University Hospital.

\section{Consent for publication}

Not applicable.

\section{Competing interests}

The authors declare that they have no competing interests related to this work.

\section{Funding}

This work was supported by the Sichuan Youth Foundation of Science of Technology (grant number: 2015JQ0026).

\section{Authors' contributions}

Y.Y., T.W.Y.H., and Z.Y.L. contributed to data collection, study concepts and study design. Y.Y., T.W.Y.H., and E.Y. contributed to literature search and meta-analysis. Y.Y. and X.M. contributed to data analysis. All of the authors contributed to data interpretation, manuscript preparation, editing and review.

\section{Acknowledgements}

The authors are grateful to Department of Gynecology and Obstetrics, West China Second University Hospital for supplying the data, and all the patients.

\section{Availability of data and materials}

The datasets used and analyzed during the current study are available from the corresponding author on reasonable request.

\section{References}

[1] Satterwhite CL, Torrone E, Meites E, Dunne EF, Mahajan R, Ocfemia MC, et al. Sexually transmitted infections among US women and men: prevalence and incidence estimates, 2008. Sex Transm Dis. 2013;40:187-193. doi: https://doi.org/10.1097/OLQ.0b013e318286bb53

[2] Zhao YQ, Zhao FH. Multi-center cross-sectional study on type-specific human papillomavirus infection among chinese women. Chinese Journal of Epidemiology|Chin J Epidemiol. 2016;36:1351-1356.

[3] Viera MH, Amini S, Huo R, Konda S, Block S, Berman B. Herpes simplex virus and human papillomavirus genital infections: new and investigational therapeutic options. Int J Dermatol. 2010;49:733-749. doi: https://doi.org/10.1111/j.1365-4632.2009.04375.x 
[4] De Sanjose S. Human papillomavirus genotype attribution in invasive cervical cancer : a retrospective cross-sectional worldwide study. Lancet Oncology. 2010;11.

[5] Serrano B, Alemany L, Tous S, Bruni L, Clifford GM, Weiss T, et al. Potential impact of a nine-valent vaccine in human papillomavirus related cervical disease. Infectious Agents \& Cancer. 2012;7:38. doi: https://doi.org/10.1186/1750-9378-7-38

[6] Plummer M, Schiffman M, Castle P, Maucort-Boulch D, Wheeler C. A 2-year prospective study of human papillomavirus persistence among women with a cytological diagnosis of atypical squamous cells of undetermined significance or low-grade squamous intraepithelial lesion. Journal of Lower Genital Tract Disease. 2008;12:152. doi: https://doi.org/10.1097/01.Igt.0000305247.00437.26

[7] Rodriguez AC, Schiffman M, Herrero R, Wacholder S, Hildesheim A, Castle PE, et al. Rapid clearance of human papillomavirus and implications for clinical focus on persistent infections. Journal of the National Cancer Institute. 2008;100:513-517. doi: https://doi.org/10.1093/jnci/djn044

[8] Elfgren K, Naucler P, Dillner J. Management of women with human papillomavirus persistence: longterm follow-up of a randomized clinical trial. American Journal of Obstetrics \& Gynecology. 2016. doi: https://doi.org/10.1016/j.ajog.2016.10.042

[9] Nayar R, Wilbur DC. The pap test and bethesda 2014. Cancer Cytopathology.59:121-132.

[10] Saslow D, Solomon D, Lawson HW, Killackey M, Kulasingam SL, Cain JM, et al. American cancer society, american society for colposcopy and cervical pathology, and american society for clinical pathology screening guidelines for the prevention and early detection of cervical cancer. American Journal of Clinical Pathology. 2012;16:147-172. doi: https://doi.org/10.1097/LGT.0b013e31824ca9d5

[11] Gage JC, Sadorra M, Lamere BJ, Kail R, Aldrich C, Kinney W, et al. Comparison of the cobas human papillomavirus (HPV) test with the hybrid capture 2 and linear array HPV DNA tests. J Clin Microbiol. 2012;50:61-65. doi: https://doi.org/10.1128/jcm.05989-11

[12] Shi HJ, Song HB, Zhao QY, Tao CX, Liu M. Efficacy and safety of combined high-dose interferon and red light therapy for the treatment of human papillomavirus and associated vaginitis and cervicitis: a prospective and randomized clinical study. Medicine. 2018;97:e12398. doi:

https://doi.org/10.1097/MD.0000000000012398

[13] Lince-Deroche N, van Rensburg C, Roseleur J, Sanusi B, Phiri J, Michelow P, et al. Costs and costeffectiveness of LEEP versus cryotherapy for treating cervical dysplasia among HIV-positive women in Johannesburg, South Africa. PloS one. 2018;13:e0203921. doi:

https://doi.org/10.1371/journal.pone.0203921

[14] Yang Y, Meng YL, Duan SM, Zhan SB, Guan RL, Yue TF, et al. REBACIN® as a noninvasive clinical interventinn for hiah-rick human nanillnmavirus nercictent infection. Int J Cancer. 2019. doi: Loading [MathJax]/jax/output/CommonHTML/fonts/TeX/fontdata.js 
https://doi.org/10.1002/ijc.32344

[15] Katundu K, Bateman AC, Pfaendler KS, Mwanahamuntu MH, Kapambwe S, Vermund SH, et al. The effect of cryotherapy on human papillomavirus clearance among HIV-positive women in Lusaka, Zambia. J Low Genit Tract Dis. 2015;19:301-306. doi: https://doi.org/10.1097/LGT.0000000000000131

[16] Gonzalez-Sanchez JL, Martinez-Chequer JC, Hernandez-Celaya ME, Barahona-Bustillos E, AndradeManzano AF. Randomized placebo-controlled evaluation of intramuscular interferon beta treatment of recurrent human papillomavirus. Obstet Gynecol. 2001;97:621-624. doi: https://doi.org/10.1016/s00297844(00)01201-1

[17] Cheng $Y X$, Jiang JH, Xue X. The influence of recombinant human $a-2 b$ interferon capsule for vaginal foams on the negative conversion rate of HPV. Journal of Practical Obstetrics \& Gynecology. 2009.

[18] Sun LL, Song ZQ. Analysis of the efficacy of recombinant human $a-2 b$ interferon capsule for vaginal foams Progress in Obstetrics and Gynecology. 2010;20:86-87.

[19] Zhong HW, Ling Y, Zhao J. The effect of Xinfuning on HPV infection in female reproductive tract. Strait Pharmaceutical Journal 2012;24:226-227.

[20] Chen R, Feng L. Clinical observation study of Paiteling on high-risk HPV infection clearance of cervical lesions after conization. The Chinese Journal of Human Sexualit. 2019;28:100-102.

[21] Chen XJ, Ye J. Clinical research on effect of vaginal local interferon application on cervical high risk human papillomavirus infection. Journal of Practical Obstetrics and Gynecology 2016;32:450-452.

[22] Rositch AF, Burke AE, Viscidi RP, Silver MI, Chang K, Gravitt PE. Contributions of recent and past sexual partnerships on incident human papillomavirus detection: acquisition and reactivation in older women. Cancer Res. 2012;72:6183-6190. doi: https://doi.org/10.1158/0008-5472.Can-12-2635

[23] Maglennon GA, Doorbar J. The biology of papillomavirus latency. Open Virol J. 2012;6:190-197. doi: https://doi.org/10.2174/1874357901206010190

[24] Theiler RN, Farr SL, Karon JM, Paramsothy P, Viscidi R, Duerr A, et al. High-risk human papillomavirus reactivation in human immunodeficiency virus-infected women: risk factors for cervical viral shedding. Obstet Gynecol. 2010;115:1150-1158. doi: https://doi.org/10.1097/AOG.0b013e3181e00927

[25] Unnop J, Xavier C, M. GS, Paulo N, Johanna P, Rowena DR-RM, et al. Natural history of progression of HPV infection to cervical lesion or clearance: analysis of the control arm of the large, randomised PATRICIA study. PloS one. 2013;8:e79260. doi: https://doi.org/10.1371/journal.pone.0079260

[26] Richardson H, Kelsall G, Tellier P, Voyer H, Abrahamowicz M, Ferenczy A, et al. The natural history of type-specific human papillomavirus infections in female university students. Cancer Epidemiol 
[27] Stewart M. 2012 Updated consensus guidelines for the management of abnormal cervical cancer screening tests and cancer precursors. Journal of Lower Genital Tract Disease. 2013;17:1-27.

[28] Katki HA, Schiffman M, Castle PE, Fetterman B, Poitras NE, Lorey T, et al. Benchmarking CIN 3+ risk as the basis for incorporating HPV and Pap cotesting into cervical screening and management guidelines. J Low Genit Tract Dis. 2013;17:28-35. doi: https://doi.org/10.1097/LGT.0b013e318285423c

[29] Winer RL HJ, Feng Q, O'Reilly S, Kiviat NB, Holmes KK, Koutsky LA. Condom use and the risk of genital human papillomavirus infection in young women. Obstetrics \& Gynecology. 2006;108:1293. doi: https://doi.org/10.1097/01.aog.0000245159.52318.a9

\section{Tables}

Table 1 The effective rate of REBACIN ${ }^{\circledR}$ in different groups. 


\begin{tabular}{|c|c|c|c|}
\hline Characteristic & & Total (N) & Effective rate (\%) \\
\hline \multirow[t]{5}{*}{ Age (years) } & $\leq 30$ & 52 & $88.46 \%(46 / 52)$ \\
\hline & $31-40$ & 107 & $71.96 \%(77 / 107)$ \\
\hline & $41-50$ & 141 & $71.63 \%(101 / 141)$ \\
\hline & $51-60$ & 55 & $76.36 \%(42 / 55)$ \\
\hline & $\geq 61$ & 9 & $66.67 \%(6 / 9)$ \\
\hline \multirow[t]{5}{*}{ HPV type } & Single-type & 229 & $66.81 \%(153 / 229)$ \\
\hline & HPV16 & 51 & $60.78 \%(31 / 51)$ \\
\hline & HPV18 & 19 & $68.42 \%(13 / 19)$ \\
\hline & Others & 159 & $68.55 \%(109 / 159)$ \\
\hline & Multi-type & 97 & $90.72 \%(88 / 97)$ \\
\hline \multirow[t]{3}{*}{ Pretreatment cytology } & NILM & 167 & $64.67 \%(108 / 167)$ \\
\hline & ASCUS & 49 & $81.63 \%(40 / 49)$ \\
\hline & LSIL & 30 & $86.67 \%(26 / 30)$ \\
\hline \multirow[t]{3}{*}{ Course of REBACIN $®$} & 1 & 294 & $71.15 \%(218 / 294)$ \\
\hline & 2 & 49 & $81.63 \%(40 / 49)$ \\
\hline & 3 & 21 & $66.67 \%(14 / 21)$ \\
\hline \multirow[t]{3}{*}{ Sex life } & With condom & 56 & $66.07 \%(37 / 56)$ \\
\hline & Without condom & 55 & $75.55 \%(41 / 55)$ \\
\hline & None & 162 & $81.48 \%(132 / 162)$ \\
\hline \multirow[t]{2}{*}{ History of interferon use } & Yes & 241 & $70.54 \%(170 / 241)$ \\
\hline & No & 31 & $77.42 \%(24 / 31)$ \\
\hline
\end{tabular}

Note. NILM: negative for intraepithelial lesion or malignancy; ASCUS: atypical squamous cells of unknown significance; LSIL: low-grade squamous intraepithelial lesion.

Table 2 Characteristics and results of the studies included the in meta-analysis. 


\begin{tabular}{lllllll}
\hline Study & Location & Design & Total & Effective & ES & $95 \%$ CI \\
\hline Gonzalez 2001[16] & Mexico & RCT & 61 & 48 & 0.787 & $0.684,0.890$ \\
Cheng 2009[17] & China & observational study & 44 & 32 & 0.727 & $0.596,0.859$ \\
Sun 2010[18] & China & observational study & 74 & 46 & 0.622 & $0.511,0.732$ \\
Zhong 2012[19] & China & observational study & 45 & 28 & 0.622 & $0.481,0.764$ \\
Shi 2018 [12] & China & RCT & 43 & 27 & 0.628 & $0.483,0.772$ \\
Cheng 2019[20] & China & observational study & 36 & 10 & 0.278 & $0.131,0.424$ \\
D+L pooled ES & & & & & 0.615 & $0.480,0.749$ \\
\hline
\end{tabular}

Note. Heterogeneity chi-squared $=33.27$ (d.f. $=5$ ); $p=0.00$.

Table 3 Factors affecting REBACIN ${ }^{\circledR}$ efficacy.

\begin{tabular}{lllll}
\hline Factors & Effective & Ineffective & OR $(95 \%$ CI $)$ & P value \\
\hline HPV type & & & $0.549(0.367,0.822)$ & $<0.01$ \\
HPV 16 & 31 & 20 & & \\
HPV 18 & 13 & 6 & & \\
Other single-type & 109 & 50 & & \\
Multi-type & 88 & 9 & & \\
Pretreatment cytology & & & & \\
NILM & 108 & 59 & & \\
ASCUS & 40 & 9 & & \\
LSIL & 26 & 4 & & \\
\hline
\end{tabular}

Note. NILM: negative for intraepithelial lesion or malignancy; ASCUS: atypical squamous cells of unknown significance; LSIL: low-grade squamous intraepithelial lesion.

Table 4 Chi-square tests for the factors influencing REBACIN ${ }^{\circledR}$ efficacy. 


\begin{tabular}{lllll}
\hline & Effective & Ineffective & P value \\
\hline HPV type & & & 20.21 & $<0.01$ \\
Multi-type & 88 & 9 & & \\
Single-type & 153 & 76 & & \\
HPV type & & & & \\
HPV 16 & 31 & 20 & & \\
HPV 18 & 13 & 6 & & \\
Other single type & 109 & 50 & & \\
Pretreatment cytology & & & & \\
NILM & 108 & 59 & & \\
ASCUS and LSIL & 66 & 11.05 & $<0.01$ \\
\hline
\end{tabular}

Note. NILM: negative for intraepithelial lesion or malignancy; ASCUS: atypical squamous cells of unknown significance; LSIL: low-grade squamous intraepithelial lesion.

\section{Figures}

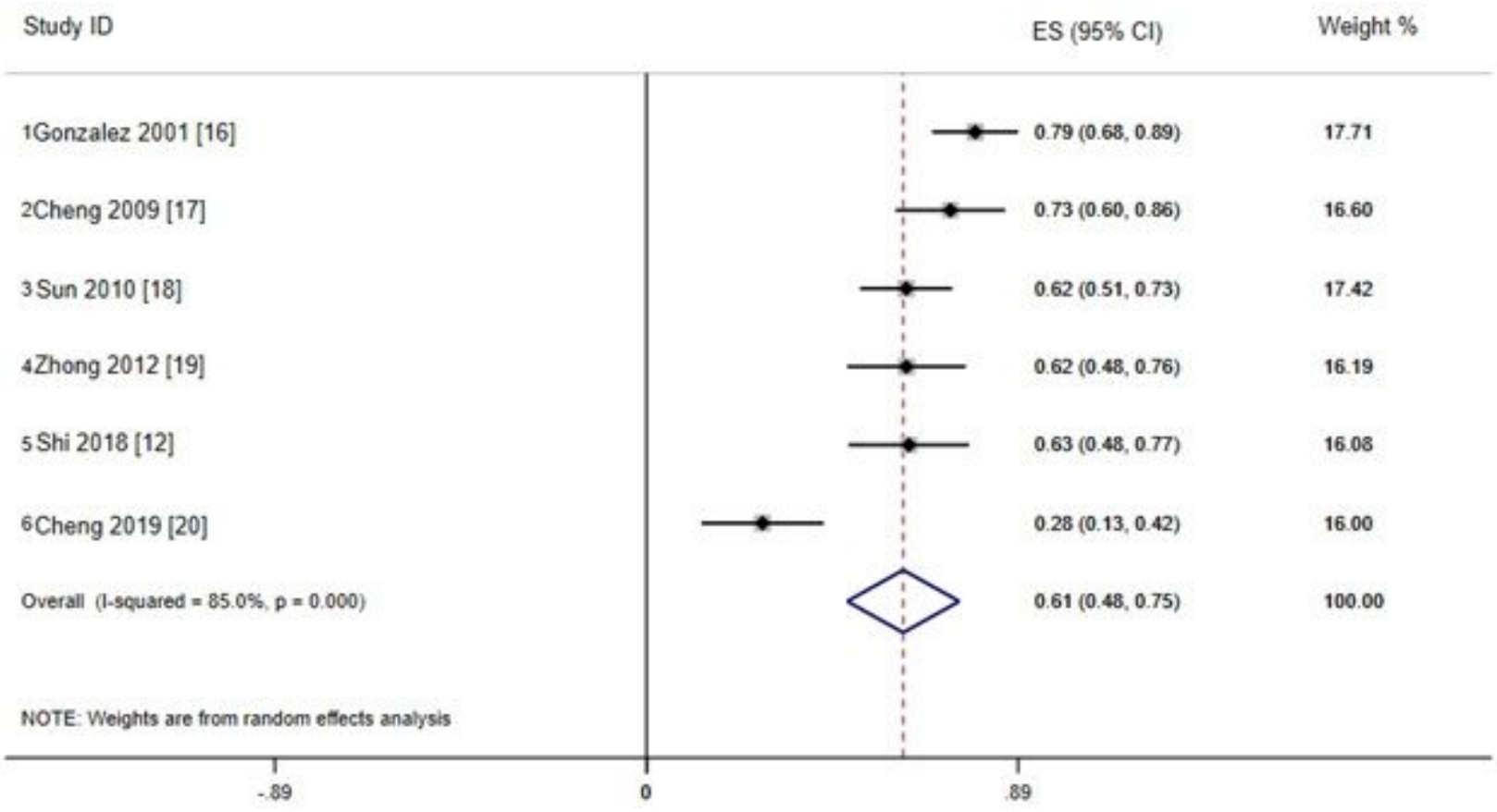

\section{Figure 1}

The effective rate of interferon: Forest plot diagram showing the pooled estimates for the effective rate of interferon. 